\title{
Documenting automated Fortran-C++ bindings with SWIG
}

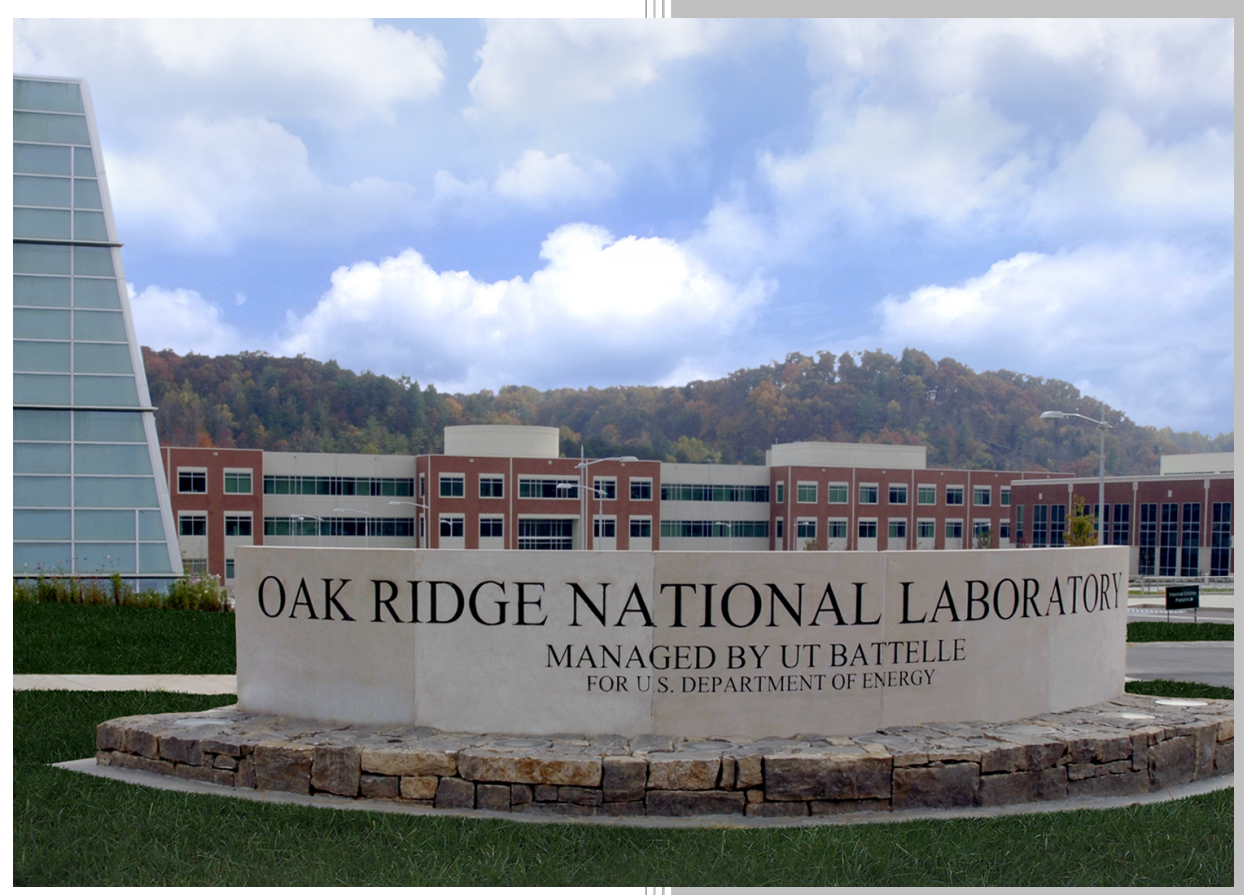

Approved for public release. Distribution is unlimited.
Andrey Prokopenko Matthew Bement Seth Johnson Katherine Evans

June 2019 


\section{DOCUMENT AVAILABILITY}

Reports produced after January 1, 1996, are generally available free via US Department of Energy (DOE) SciTech Connect.

Website: www.osti.gov/

Reports produced before January 1, 1996, may be purchased by members of the public from the following source:

National Technical Information Service

5285 Port Royal Road

Springfield, VA 22161

Telephone: 703-605-6000 (1-800-553-6847)

TDD: $703-487-4639$

Fax: 703-605-6900

E-mail: info@ntis.gov

Website: http://classic.ntis.gov/

Reports are available to DOE employees, DOE contractors, Energy Technology Data Exchange representatives, and International Nuclear Information System representatives from the following source:

Office of Scientific and Technical Information

PO Box 62

Oak Ridge, TN 37831

Telephone: 865-576-8401

Fax: 865-576-5728

E-mail: report@osti.gov

Website: http://www.osti.gov/contact.html

This report was prepared as an account of work sponsored by an agency of the United States Government. Neither the United States Government nor any agency thereof, nor any of their employees, makes any warranty, express or implied, or assumes any legal liability or responsibility for the accuracy, completeness, or usefulness of any information, apparatus, product, or process disclosed, or represents that its use would not infringe privately owned rights. Reference herein to any specific commercial product, process, or service by trade name, trademark, manufacturer, or otherwise, does not necessarily constitute or imply its endorsement, recommendation, or favoring by the United States Government or any agency thereof. The views and opinions of authors expressed herein do not necessarily state or reflect those of the United States Government or any agency thereof. 
Computational Sciences and Engineering Division

DOCUMENTING AUTOMATED FORTRAN-C++ BINDINGS WITH SWIG

Andrey Prokopenko (PI) (Oak Ridge National Laboratory)

Matthew Bement (Los Alamos National Laboratory)

Seth Johnson (Oak Ridge National Laboratory)

Katherine Evans (Oak Ridge National Laboratory)

Date Published: June 2019

Prepared by

OAK RIDGE NATIONAL LABORATORY

Oak Ridge, TN 37831-6283

managed by

UT-Battelle, LLC

for the

US DEPARTMENT OF ENERGY

under contract DE-AC05-00OR22725 


\section{CONTENTS}

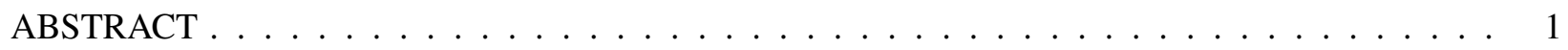

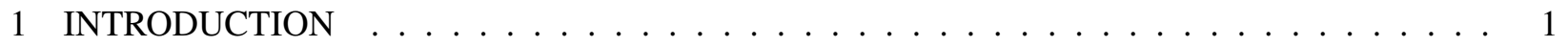

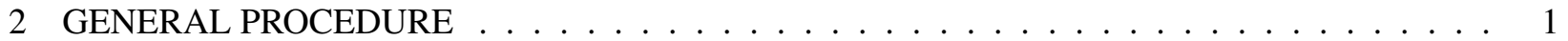

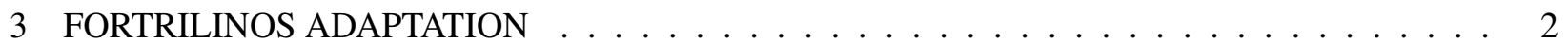

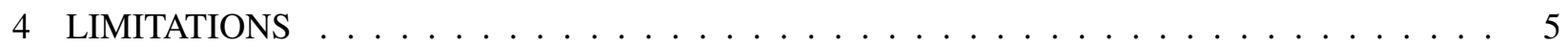

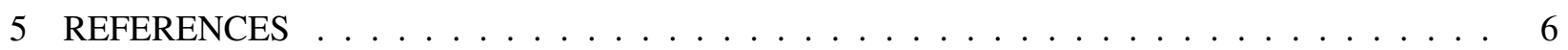




\begin{abstract}
A new SWIG/Fortran tool introduced in Johnson et al. [to appear] automatically generates native Fortran 2003 interfaces to $\mathrm{C}$ and $\mathrm{C}++$ libraries. This allows a seamless integration of existing $\mathrm{C}++$ libraries into the Fortran applications. However, using the generated interfaces is complicated by the lack of appropriate documentation. In this report, we document a way to automatically port the existing Doxygen documentation of the $\mathrm{C}++$ libraries to Fortran. We use ForTrilinos library as our target application, and discuss the scope and limitations of this approach.
\end{abstract}

\title{
1 INTRODUCTION
}

Doxygen is both a tool and a specification for writing documentation for software. The documentation is written within code comments, and thus is relatively easy to maintain. The documented code is run through the doxygen tool to produce output in one of the available formats, such as HTML, ETEX, etc. Many large scientific projects, such as Trilinos Heroux et al. [2003], rely on Doxygen to document their application programming interface (API), hosting the generated HTML output on a website for easy access by the users.

Doxygen supports many programming languages. Of particular importance, it supports both $\mathrm{C}++$ and Fortran. When running doxygen, it is possible to produce the output in language-agnostic form, i.e., XML format. Thus, if one is able to write a tool to convert Doxygen XML output back to a specific language, and to insert it in the right place of the generated code, it would be possible to translate comments from one language to the other.

In this report, we discuss the steps required to insert Doxygen documentation into the automatically generated Fortran interface wrappers for an existing $\mathrm{C}++$ library.

\section{GENERAL PROCEDURE}

In this Section, we describe the steps to automatically port documentation from a $\mathrm{C}++$ header to an automatically generated Fortran interface wrapper.

\section{Step 1. Produce language-agnostic XML output}

The first step is to use Doxygen to generate XML output. This is done by runnning the doxygen tool on the relevant $\mathrm{C}++$ library headers. As an input, doxygen takes in a Doxygen configuration file (Doxyfile). A default Doxyfile could be generated by running

$\$$ doxygen $-\mathrm{g}$

In order to produce XML output, the option GENERATE_XML in the Doxyfile must be set to YES.

\section{Step 2. Convert XML output to SWIG docstrings}

In order for the documentation be attached in the right places in the generated output later, it must first be converted to a SWIG-readable format called docstrings. A docstring is a SWIG code snippet containing the function name and its documentation, including the input arguments. For example, 


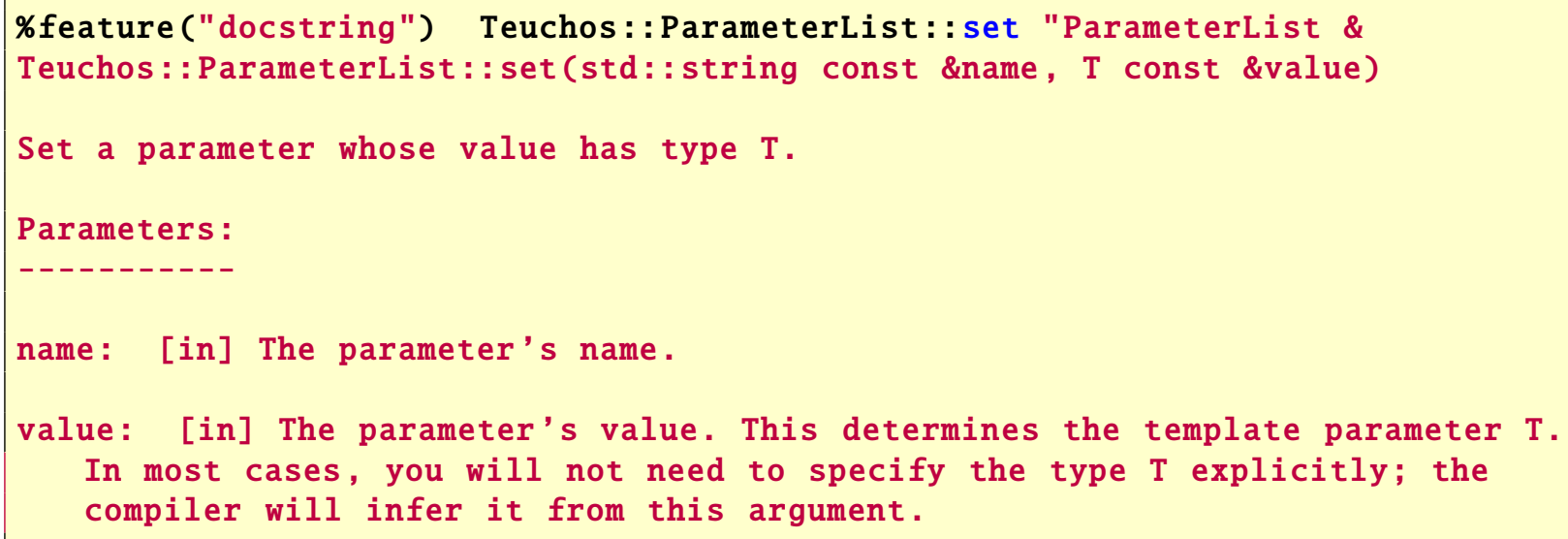

This is done by running a Python script doxy2swig.py (available as part of the Trilinos' package PyTrilinos) as follows:

\$ doxy2swig.py doxygen_output.xml swig_docstrings.i

Here, doxygen_output. $\mathrm{xml}$ is the Doxygen XML output produced by the first step, and swig_docstrings. $i$ is the resulting SWIG file containing docstrings for all the functions in the index.xml.

\section{Step 3. Include SWIG docstrings file into SWIG interface file}

This file of docstrings must then be included in the SWIG interface file like this:

\%include "swig_docstrings.i"

... rest of SWIG file...

When SWIG is run, the documentation strings will be automatically inserted in the appropriate places in the wrapper code in the form of native language comments.

\section{Step 4. Run Doxygen on the produced Fortran wrapper files}

The last step is to run doxygen again on the produced wrapper file to generate the documentation in the desired format.

\section{FORTRILINOS ADAPTATION}

In this Section, we describe the adaptation of the general approach described in Section 2 to ForTrilinos. For reasons documented in Section 4, we require doxygen version 1.8.15 or above.

ForTrilinos provides interfaces for several Trilinos packages (Belos, Teuchos, Tpetra) and a general linear and nonlinear solver interfaces. We will use Tpetra to describe the documentation generation.

As discussed in Section 2, the first step is to generate Doxygen XML documentation. In order to automatically pick up the location of the original Trilinos Tpetra headers, ForTrilinos carries Doxyfile_tpetra.in file with the following code: 
INPUT = QPROJECT_SOURCE_DIR@/packages/tpetra/classic/src I

@PROJECT_SOURCE_DIR@/packages/tpetra/classic/NodeAPI ।

QPROJECT_SOURCE_DIR@/packages/tpetra/core/src ।

QPROJECT_SOURCE_DIR@/packages/tpetra/kernels/src ।

QPROJECT_SOURCE_DIR@/packages/tpetra/tsqr/src

During ForTrilinos configuration, the @PROJECT_SOURCE_DIR@ macro is automatically replaced by the correct location of the Trilinos source directory. We note here that we used this approach instead of specifying a relative (to ForTrilinos) path as the later does not properly work with symbolic links.

The Doxyfile_tpetra.in is also modified to have

EXTRACT_PRIVATE $=$ YES

EXTRACT_ALL $=$ YES

This step produces a file tpetra_dox. $i$ containing the appropriate docstrings, and places it in the ForTrilinos/src/tpetra/src directory, containing other SWIG files used to generate Tpetra interfaces. The main file, fortpetra.i, contains the following line

\%include "tpetra_dox.i"

in order to automatically pick up docstrings with SWIG.

The ForTrilinos wrapper files are generated only in developer mode (enabled with

-DForTrilinos_ENABLE_DeveloperMode=0N). The generated file, fortpetra.F90 in this case, will contain the required documentation, a snippet of which is shown below:

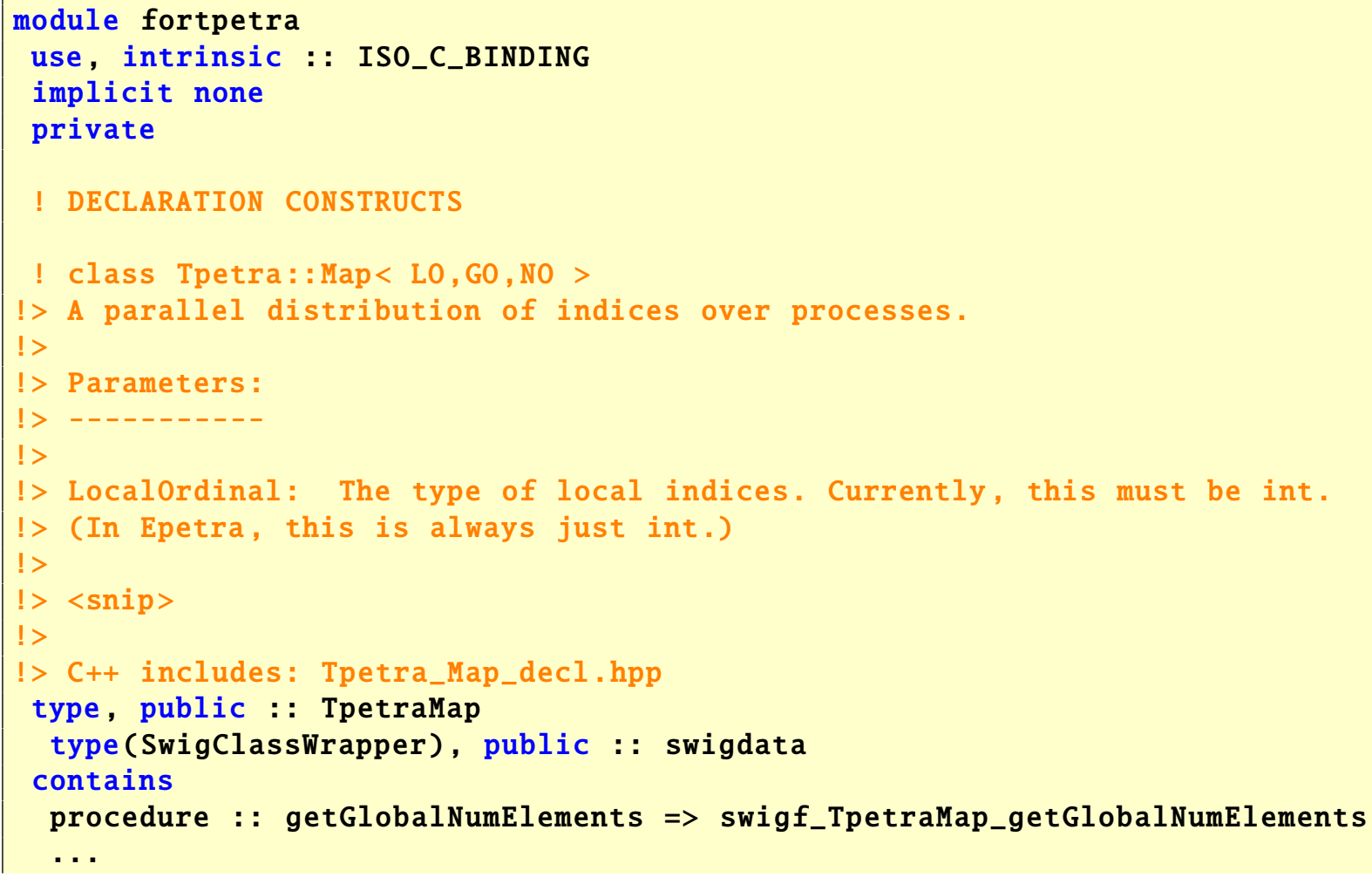




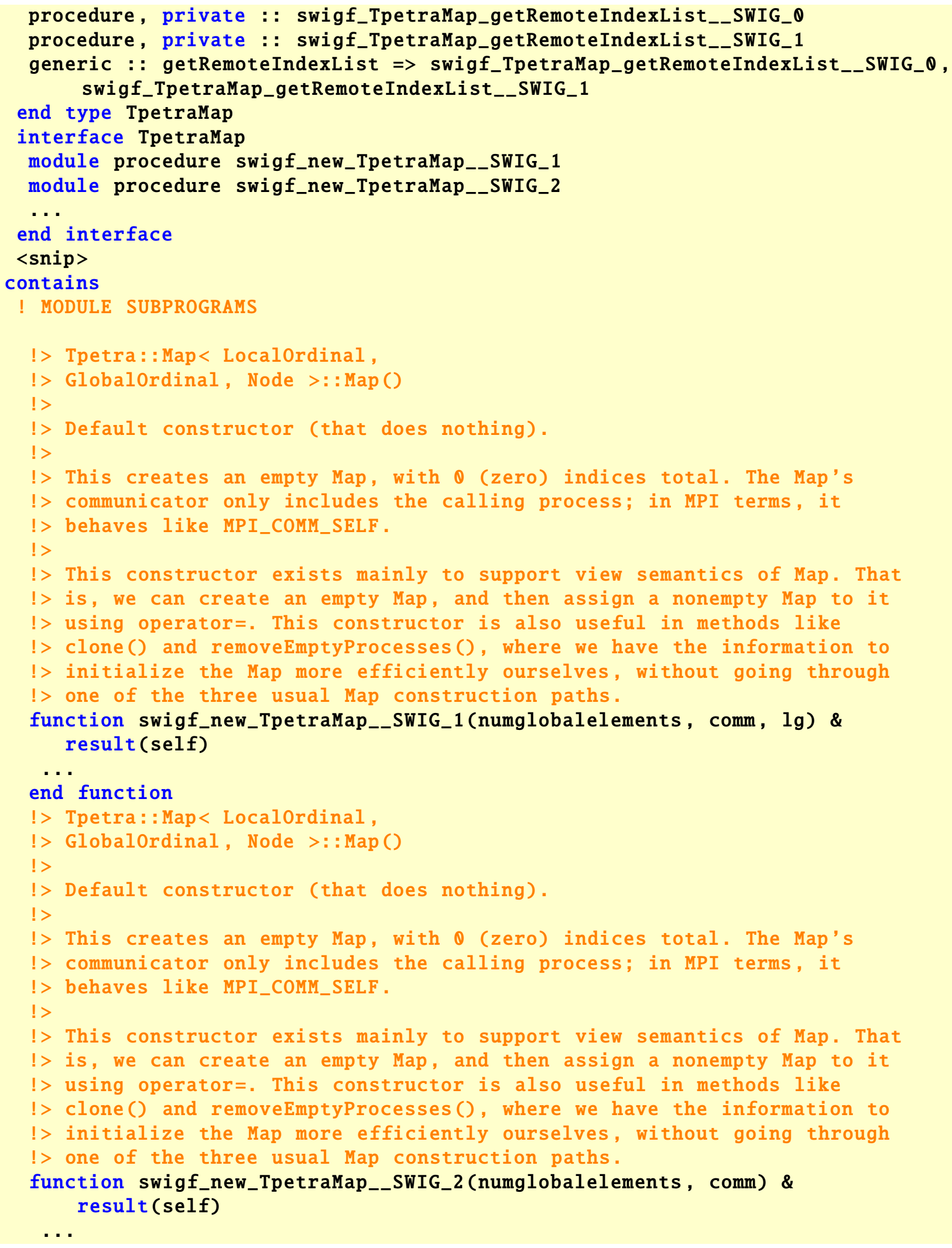




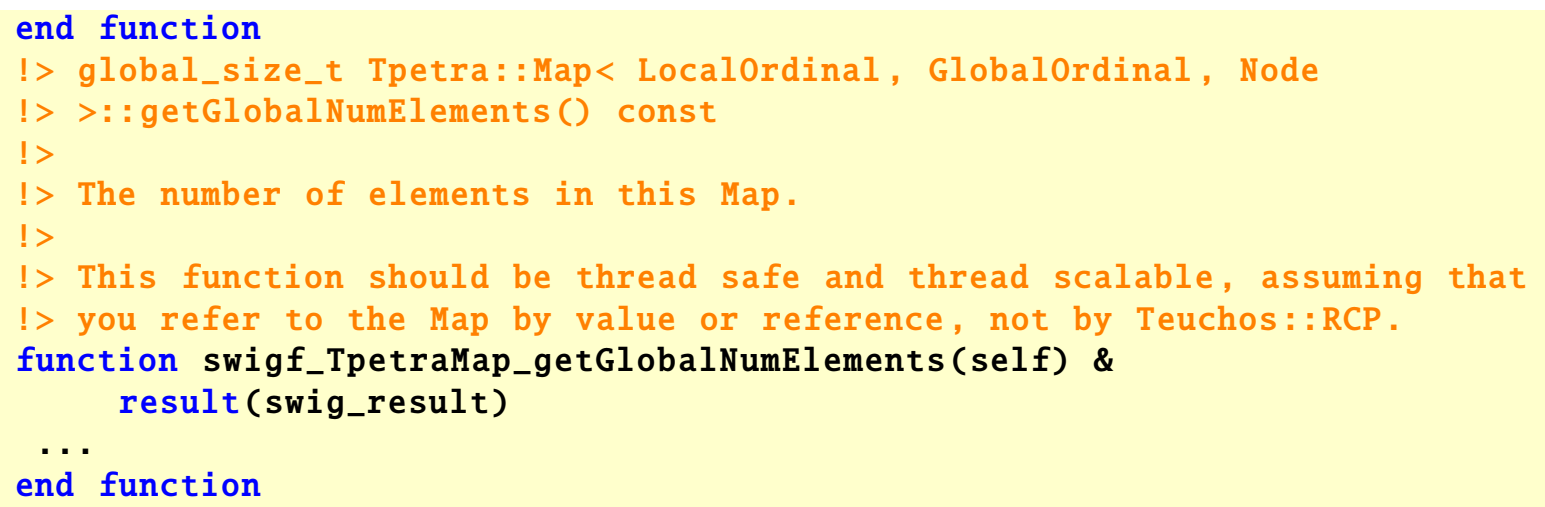

As we can see, the generated documentation is inserted into the Fortran wrapper file in correct places. However, due to limitations described in Section 4, the documentation for the constructors is repeated.

The final run of doxygen on the generated F90 file produces the results as shown in Figures 1 and 2.

\section{LIMITATIONS}

The described procedure works well in many situations. However, it also has significant drawbacks that are hard to overcome.

Specifically, for $\mathrm{C}++$ to Fortran documentation translation, we encountered:

\section{- The docstrings are simply copied from the XML documentation}

Often, the $\mathrm{C}++$ documentation uses $\mathrm{C}++$ syntax and terminology, references $\mathrm{C}++$ classes and methods. Ideally, such syntax should be translated into the wrapper language syntax, so that all of the documentation visible to the user of a package would be in native format.

\section{- Generic procedures are handled incorrectly}

Fortran generic procedures pose a challenge on multiple fronts. First, there are challenges related to Doxygen itself. For example, note the getremoteindexlist generic of the Tpetramap interface in Figure 1. If one follows the link to swigf_tpetramap_getremoteinexlist_swig_ $\theta$, there is essentially no documentation, as shown in Figure 3.

To access the documentation for this procedure, one has to refer back to the top level fortpetra interface documentation, navigate to the Functions/Subroutines section and then follow the link to swigf_tpetramap_getremoteinexlist_swig_ $\theta$, where one finds the expected documentation, as is shown in three images shown in Figure 4.

When one does access the documentation for the generic procedures in this manner, the documentation is identical for different function signatures, as is also seen in Figure 2. This is due to how doxy2swig handles the different argument lists. It is not currently known if modifications to doxy2 swig could address this limitation, and if so, if SWIG allows docstrings to be associated with function signatures in addition to function names. 
We also note that only recent versions of Doxygen seem to work. The recommended version is 1.8 .15 or above. For example, we found that Doxygen 1.8.5 does not link the documentation of the binding name function to the procedure name.

\section{REFERENCES}

Michael Heroux et al. An overview of Trilinos. Technical Report SAND2003-2927, Sandia National Laboratories, 2003.

Seth R. Johnson, Andrey Prokopenko, and Katherine J. Evans. Automated Fortran-C++ bindings for large-scale scientific applications. Computing in Science $\mathcal{E}$ Engineering, to appear. 


\section{ForTrilinos}

$\begin{aligned} & \text { Main Page } \\ & \text { fortpetra }\rangle \text { tpetramap }\rangle\end{aligned}$
fortpetra::tpetramap Interface Reference
Data Types List ₹

A parallel distribution of indices over processes. More.

Public Member Functions

\begin{tabular}{|c|c|}
\hline procedure & release => swigf_release_tpetramap \\
\hline procedure & isonetoone => swigf_tpetramap_isonetoone \\
\hline procedure & getglobalnumelements => swigf_tpetramap_getglobalnumelements \\
\hline procedure & getnodenumelements => swigf_tpetramap_getnodenumelements \\
\hline procedure & getminlocalindex => swigf_tpetramap_getminlocalindex \\
\hline procedure & getmaxlocalindex => swigf_tpetramap_getmaxlocalindex \\
\hline procedure & getminglobalindex => swigf_tpetramap_getminglobalindex \\
\hline procedure & getmaxglobalindex => swigf_tpetramap_getmaxglobalindex \\
\hline procedure & getminallglobalindex => swigf_tpetramap_getminallglobalindex \\
\hline procedure & getmaxallglobalindex => swigf_tpetramap_getmaxallglobalindex \\
\hline procedure & getlocalelement => swigf_tpetramap_getlocalelement \\
\hline procedure & getglobalelement => swigf_tpetramap_getglobalelement \\
\hline procedure & getnodeelementlist => swigf_tpetramap_getnodeelementlist \\
\hline procedure & isnodelocalelement => swigf_tpetramap_isnodelocalelement \\
\hline procedure & isnodeglobalelement => swigf_tpetramap_isnodeglobalelement \\
\hline procedure & isuniform => swigf_tpetramap_isuniform \\
\hline procedure & iscontiguous => swigf_tpetramap_iscontiguous \\
\hline procedure & isdistributed => swigf_tpetramap_isdistributed \\
\hline procedure & iscompatible => swigf_tpetramap_iscompatible \\
\hline procedure & issameas => swigf_tpetramap_issameas \\
\hline procedure & locallysameas $=>$ swigf_tpetramap_locallysameas \\
\hline procedure & islocallyfitted => swigf_tpetramap_islocallyfitted \\
\hline procedure & getcomm => swigf_tpetramap_getcomm \\
\hline procedure & description => swigf_tpetramap_description \\
\hline procedure & removeemptyprocesses => swigf_tpetramap_removeemptyprocesses \\
\hline procedure & replacecommwithsubset => swigf_tpetramap_replacecommwithsubset \\
\hline generic & assignment => swigf_tpetramap_op_assign__ \\
\hline generic & getremoteindexlist => swigf_tpetramap_getremoteindexlist_swig_0, swigf_tpetramap_getremoteindexlist_swig_1 \\
\hline type(tpetramap) function & $\begin{array}{l}\text { swigf_new_tpetramap_swig_1 (numglobalelements, comm, Ig) } \\
\text { Tpetra:Map< LocalOrdinal, GlobalOrdinal, Node >:Map() More... }\end{array}$ \\
\hline type(tpetramap) function & $\begin{array}{l}\text { swigf_new_tpetramap_swig_2 (numglobalelements, comm) } \\
\text { Tpetra::Map< LocalOrdinal, GlobalOrdinal, Node >:Map0 More... }\end{array}$ \\
\hline type(tpetramap) function & $\begin{array}{l}\text { swigf_new_tpetramap_swig_4 (numglobalelements, numlocalelements, comm) } \\
\text { Tpetra::Map< LocalOrdinal, GlobalOrdinal, Node >:Map } 0 \text { More... }\end{array}$ \\
\hline type(tpetramap) function & $\begin{array}{l}\text { swigf_new_tpetramap_swig_7 (numglobalelements, indexlist, comm) } \\
\text { Tpetra::Map< LocalOrdinal, GlobalOrdinal, Node >:-Map0 More... }\end{array}$ \\
\hline type(tpetramap) function & $\begin{array}{l}\text { swigf_new_tpetramap_swig_8 } 0 \\
\text { Tpetra:Map<LocalOrdinal, GlobalOrdinal, Node >:Map0 More... }\end{array}$ \\
\hline integer(tpetralookupstatus) function & $\begin{array}{l}\text { swigf_tpetramap_getremoteindexlist_swig_0 (self, gidlist, nodeidlist, lidlist) } \\
\text { LookupStatus Tpetra:Map<LocalOrdinal, GlobalOrdinal, Node >:-getRemotelndexList(const Teuchos::ArrayView< const } \\
\text { GlobalOrdinal > \&GIDList, const Teuchos::ArrayView< int > \&nodelDList) const. More... }\end{array}$ \\
\hline \multicolumn{2}{|c|}{ • swigf_new_tpetramap_swig_1() } \\
\hline \multicolumn{2}{|c|}{$\begin{array}{ll}\text { type(tpetramap) function fortpetra:swigf_new_tpetramap_swig_1 ( integer(c_long), intent(in) } & \begin{array}{l}\text { numglobalelemen } \\
\text { class(teuchoscomm), intent(in) } \\
\text { comm, } \\
\text { integer(tpetralocalglobal), intent(in) } \lg \end{array} \\
\text { ) } & \end{array}$} \\
\hline \multicolumn{2}{|c|}{ Tpetra::Map< LocalOrdinal, GlobalOrdinal, Node >:Map0) } \\
\hline \multicolumn{2}{|l|}{ Default constructor (that does nothing). } \\
\hline This creates an empty Map, with 0 (ze & ero) indices total. The Map's communicator only includes the calling process; in MPI terms, it behaves like MPI_COMM_SELF. \\
\hline $\begin{array}{l}\text { This constructor exists mainly to supp } \\
\text { constructor is also useful in methods I } \\
\text { going through one of the three usual } \mathrm{N}\end{array}$ & $\begin{array}{l}\text { port view semantics of Map. That is, we can create an empty Map, and then assign a nonempty Map to it using operator=. This } \\
\text { like clone } \text { ( and removeEmptyProcesses (), where we have the information to initialize the Map more efficiently ourselves, without } \\
\text { Map construction paths. }\end{array}$ \\
\hline
\end{tabular}

Figure 1. Generated Doxygen documentation for TpetraMap. 


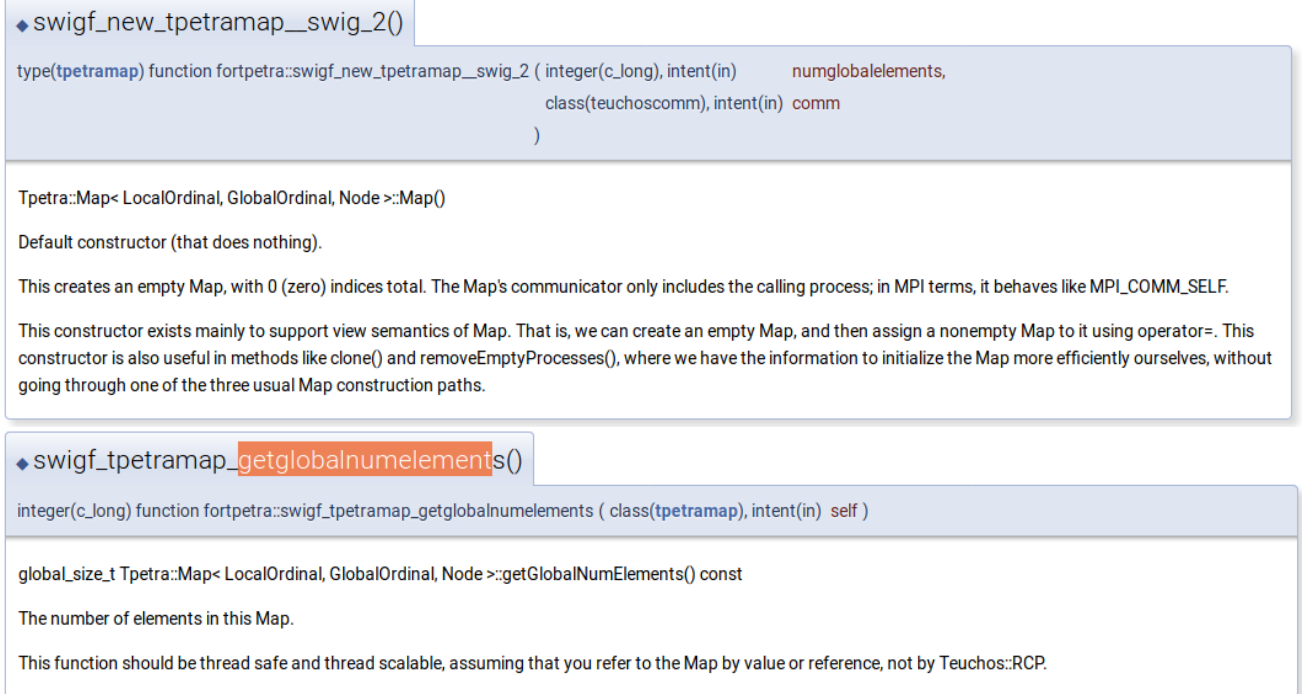

Figure 2. Generated Doxygen documentation for TpetraMap.

- swigf_tpetramap_getremoteindexlist_swig_0()

procedure, private fortpetra::tpetramap::swigf_tpetramap_getremoteindexlist_swig_0( ) private

Figure 3. Incorrect Doxygen output for TpetraMap generic. 


\begin{tabular}{|c|c|}
\hline subroutine & fortpetra::swig_string_to_chararray (string, chars, wrap) \\
\hline subroutine, public & fortpetra::setcombinemodeparameter (plist, paramname) \\
\hline subroutine & fortpetra::swig_chararray_to_string (wrap, string) \\
\hline character(kind=c_char, len=:) function, allocatable, public & fortpetra: combinemodetostring (combinemode) \\
\hline type(tpetramap) function & $\begin{array}{l}\text { fortpetra: :swigf_new_tpetramap_swig_1 (numglobalelements, comm, Ig) } \\
\text { Tpetra::Map< LocalOrdinal, GlobalOrdinal, Node >::Map() More... }\end{array}$ \\
\hline type(tpetramap) function & $\begin{array}{l}\text { fortpetra::swigf_new_tpetramap_swig_2 (numglobalelements, comm) } \\
\text { Tpetra::Map<LLocalOrdinal, GlobalOrdinal, Node >::Map() More... }\end{array}$ \\
\hline type(tpetramap) function & $\begin{array}{l}\text { fortpetra::swigf_new_tpetramap_swig_4 (numglobalelements, } \\
\text { numlocalelements, comm) } \\
\text { Tpetra::Map< LocalOrdinal, GlobalOrdinal, Node >::Map() More... }\end{array}$ \\
\hline type(tpetramap) function & $\begin{array}{l}\text { fortpetra::swigf_new_tpetramap_swig_7 (numglobalelements, indexlist, comm) } \\
\text { Tpetra::Map< Localordinal, GlobalOrdinal, Node }>:: \text { Map() More... }\end{array}$ \\
\hline type(tpetramap) function & $\begin{array}{l}\text { fortpetra::swigf_new_tpetramap_swig_8 () } \\
\text { Tpetra::Map< LocalOrdinal, GlobalOrdinal, Node }>: \text { :Map() More... }\end{array}$ \\
\hline
\end{tabular}

integer(tpetralookupstatus) function fortpetra: swigf_tpetramap_getremoteindexlist_swig_o (self, gidlist, nodeidlist, lidlist)

LookupStatus Tpetra::Map< LocalOrdinal, GlobalOrdinal, Node $>$ ::getRemotelndexList(const Teuchos: ArrayView< const GlobalOrdinal > \&GIDList, const Teuchos: ArrayView < int $>$ \&nodelDList) const. More...

\section{- swigf_tpetramap_getremoteindexlist_swig_0()}

integer(tpetralookupstatus) function

fortpetra::swigf_tpetramap_getremoteindexlist_swig_0

( class(tpetramap), intent(in) self,

integer(c_long_long), dimension(:), target gidlist,

integer(c_int), dimension(:), target nodeidlist,

integer(c int), dimension(:), target lidlist

)

LookupStatus Tpetra::Map< LocalOrdinal, GlobalOrdinal, Node > : getRemoteIndexList(const Teuchos::ArrayView< const GlobalOrdinal > \&GIDList, const Teuchos: :ArrayView< int > \&nodelDList) const.

Return the process ranks for the given global indices.

This method must always be called as a collective over all processes in the Map's communicator. For a distributed noncontiguous Map, this operation requires communication.

GIDList: [in] List of global indices for which to find process ranks and local indices. These global indices need not be owned by the calling process. Indeed, they need not be owned by any process.

nodeIDList: [out] List of process ranks corresponding to the given global indices. If a global index does not belong to any process, the resulting process rank is -1 .

nodeIDList.size ()$==$ GIDList.size ()

IDNotPresent indicates that for at least one global index, we could not find the corresponding process rank. Otherwise, return AllIDsPresent.

For a distributed noncontiguous Map, this operation requires communication. This is crucial technology used in Export, Import, CrsGraph, and Crsmatrix.

Figure 4. Correct Doxygen output for TpetraMap generic. 